\title{
tRNAs as a Driving Force of Genome Evolution in Yeast
}

\author{
Ana Rita Guimarães, Inês Correia, Inês Sousa, Carla Oliveira, Gabriela Moura, \\ Ana Rita Bezerra* and Manuel A. S. Santos*
}

Department of Medical Sciences, Institute of Biomedicine - iBiMED, University of Aveiro, Aveiro, Portugal

OPEN ACCESS

Edited by:

Omar Orellana,

University of Chile, Chile

Reviewed by:

Francisco A. Cubillos,

University of Santiago, Chile

Cécile Neuvéglise,

Institut National de la Recherche

Agronomique, Centre Montpellier,

France

*Correspondence: Ana Rita Bezerra armbezerra@ua.pt

Manuel A. S. Santos msantos@ua.pt

Specialty section:

This article was submitted to Microbial Physiology and Metabolism,

a section of the journal

Frontiers in Microbiology

Received: 26 November 2020

Accepted: 01 February 2021

Published: 11 March 2021

Citation:

Guimarães AR, Correia I, Sousa I, Oliveira C, Moura G, Bezerra AR and

Santos MAS (2021) tRNAs as

a Driving Force of Genome Evolution in Yeast. Front. Microbiol. 12:634004.

doi: 10.3389/fmicb.2021.634004
Transfer RNAs (tRNAs) are widely known for their roles in the decoding of the linear mRNA information into amino acid sequences of proteins. They are also multifunctional platforms in the translation process and have other roles beyond translation, including sensing amino acid abundance, interacting with the general stress response machinery, and modulating cellular adaptation, survival, and death. In this mini-review, we focus on the emerging role of tRNA genes in the organization and modification of the genomic architecture of yeast and the role of tRNA misexpression and decoding infidelity in genome stability, evolution, and adaption. We discuss published work showing how quickly tRNA genes can mutate to meet novel translational demands, how tRNAs speed up genome evolution, and how tRNA genes can be sites of genomic instability. We highlight recent works showing that loss of tRNA decoding fidelity and small alterations in tRNA expression have unexpected and profound impacts on genome stability. By dissecting these recent evidence, we hope to lay the groundwork that prompts future investigations on the mechanistic interplay between tRNAs and genome modification that likely triggers genome evolution.

Keywords: tRNA, yeast, chromatin structure, chromosome architecture, genome evolution, genomic instability

\section{INTRODUCTION}

Transfer RNAs (tRNAs) are short non-coding RNAs, approximately 70 to 100 bases long, that play essential roles in translation by linking mRNA codons to their corresponding amino acids, following a set of decoding rules established by the genetic code. They do so by base pairing their anticodon triplets with mRNA codon triplets in the ribosome decoding center and transferring the amino acid attached to its $3^{\prime}$-end in the ribosomal peptidyl transferase center (Phizicky and Hopper, 2010). This is a critical cellular process that requires tight control of tRNA gene expression, tRNA maturation, tRNA charging, and turnover (Chan and Lowe, 2016). In actively dividing yeast cells, tRNAs represent approximately $15 \%$ of total RNA (Warner, 1999), indicating that their genes (tDNAs) are highly transcribed. In general, tDNAs are nucleosome free and are flanked by strongly positioned nucleosomes (Yuan et al., 2005; Cole et al., 2012). Their transcription is mediated by RNA polymerase III (Pol III) upon recruitment to the promoter by the transcription factors TFIIIC and TFIIIB. TFIIIC binds to the internal A-box and B-box promoter elements and helps recruit the multi-subunit factor TFIIIB to AT-rich sequences upstream of the transcription start site, forming a 
highly stable TFIIIB-DNA complex that participates in multiple rounds of Pol III recruitment and initiation (Schramm and Hernandez, 2002). In Saccharomyces cerevisiae, the cellular concentration of each tRNA is directly proportional to its gene copy number (Percudani et al., 1997). This is particularly important because translation efficiency is described as the degree to which the tRNA pool can accommodate the transcriptome, thus affecting protein production and accuracy (dos Reis et al., 2004). This interplay is fine-tuned by codon usage, which is under selective pressure and show variation across budding yeast species (LaBella et al., 2019). Yet tRNAs have other non-canonical roles in the biological theater beyond their role as adaptors in protein synthesis (reviewed in Raina and Ibba, 2014 and Su et al., 2020). For example, tDNAs have roles in chromatin organization and gene regulation and are sites for binding of numerous chromatin proteins, including the architectural structural maintenance of chromosomes (SMC) proteins, nuclear pore proteins, chromatin remodelers, and histone modifiers (D’Ambrosio et al., 2008; Su et al., 2020).

The $275 \mathrm{tDNAs}$ present in the yeast $S$. cerevisiae genome are dispersed throughout the linear maps of the 16 chromosomes. Fluorescence in situ hybridization microscopy (FISH) showed that $\mathrm{tDNAs}$ cluster at the outer periphery of the nucleolus in a microtubule-dependent manner and or adjacent to centromeres (Thompson et al., 2003). This happens with the assistance of condensing complexes bound at each tDNA gene locus (Haeusler et al., 2008) and requires substantial rearrangements of the genome topology. Whether individual tDNA associations play a role in genome organization is still poorly understood. We review below recent works on how tDNAs and related Pol III promoter elements function as boundary elements that limit chromatin domains (Donze and Kamakaka, 2001), how they work as barriers to DNA replication fork progression, and how they contribute to the formation of genomic fragile sites (Pryce et al., 2009). Beyond their role in the three-dimensional and functional organization of the genome, this review also describes how changes in the tRNA pool can drive genome evolution in fungi.

\section{ROLES OF TRNA GENES IN CHROMATIN REMODELING AND GENOME ORGANIZATION}

The three-dimensional organization of the genome can promote long-range genomic rearrangements between interacting loci whose associated chromatin and transcriptional states can be selected through evolution (Bagadia et al., 2016). In yeast, tRNA genes have been implicated in the spatial organization of the genome by acting as barrier elements and by regulating chromatin structure (Donze and Kamakaka, 2001; Noma et al., 2006; Simms et al., 2008; Iwasaki et al., 2010; Hamdani et al., 2019). Evidence that tDNAs can hamper silenced chromatin domains from invading active domains was first obtained in S. cerevisiae (Donze and Kamakaka, 2001; Simms et al., 2004), where the deletion of a Thr-tRNA $A G U$ gene at the transcriptionally silent HMR mating-type locus resulted in the spread of silencing and consequent repression of the GIT1 gene on chromosome III (Donze and Kamakaka, 2001). A GlntRNA $_{U U G}$ gene has also been shown to block silencing at the S. cerevisiae rDNA locus (RDN1) (Biswas et al., 2009). Insulator activity was similarly shown in Schizosaccharomyces pombe, where deletion of a centromeric Ala-tRNA gene led to the spread of pericentromeric heterochromatin and gene silencing (Scott et al., 2006). The precise mechanisms by which tDNAs exert their barrier function remain largely unexplored; however, the assembly of the complete Pol III transcription apparatus does seem to be required for barrier function (Donze and Kamakaka, 2001; Scott et al., 2006; Biswas et al., 2009). Mutations in internal Thr-tRNA promoter elements, A-box or B-box, at the HMR locus led to deficiencies of TFIIIC and TFIIIB assembly, resulting in the loss of barrier function in S. cerevisiae (Donze and Kamakaka, 2001). Furthermore, yeast cohesin complex mutants ( $\Delta s m c 1$ and $\Delta s m c 3)$ have impaired tDNA-mediated insulator function (Donze et al., 1999).

A study by Duan et al. (2010) mapped cis- and transinteractions across the entire genome in $S$. cerevisiae and showed that physical interactions among tDNAs are significantly enriched and that they largely co-localize into clusters associated with the nucleolus or centromeres (Duan et al., 2010). Other studies, using DNA FISH, also showed that some tRNA genes cluster together near centromeres (Thompson et al., 2003). Furthermore, microscopic observations and genome-wide mapping of physical interactions show the co-localization of TFIIIC, cohesins, and other structural proteins at tDNA physical domain borders, suggesting that these insulators are critical players in chromosome folding and organization in the yeast nucleus. Recently, Hamdani et al. (2019) devised a strategy to tackle this topic. They eliminated the internal promoter elements (A-box and B-box) of two tDNAs on the left arm and eight tDNAs on the right arm of chromosome III in S. cerevisiae to generate a "tDNA-less" chromosome where binding of transcription factors TFIIIC and TFIIIB and chromatin proteins was abrogated. This allowed the detailed characterization of chromatin packaging, folding, and nuclear dynamics of chromosome III. Using various approaches, such as MNase-seq, ChIP-seq, RNA-seq, and fluorescence microscopy co-localization analysis, authors showed that (1) tDNA loss affects chromatin structure by disrupting the precise nucleosome positioning outside tDNAs; (2) tDNAs are essential to recruitment of cohesins and condensins; and (3) tDNAs influence centromere clustering, which in turn affects nuclear architecture. Lastly, as in previous studies (Donze and Kamakaka, 2001; Simms et al., 2004; Biswas et al., 2009), loss of tDNAs alters the long-range interactions of the silenced HML and HMR loci of chromosome III, leading to alterations in gene silencing (Hamdani et al., 2019).

The discovery of tDNA insulator function in yeast along with the recent advances in uncovering their involvement in the functional and spatial organization of the genome is particularly relevant because they provide a framework for future studies in this field. Furthermore, tDNA insulator functions seem to be conserved from yeast to humans (Raab et al., 2012), and their activities appear to be associated with a significant number 
of protein complexes whose actions and regulation remain to be determined.

\section{tRNA GENES, R-LOOPS, AND TRANSPOSABLE ELEMENTS}

tDNAs are often located near naturally occurring genomic fragile sites, and genome-wide studies in S. cerevisiae have detected R-loops at tRNA genes, along with other Pol III transcribed genes (Chan et al., 2014; El Hage et al., 2014; Wahba et al., 2016; Yeung and Smith, 2020). The replication machinery naturally slows down at tDNAs, and DNA helicases must take action to promote the progression of the replication fork (Ivessa et al., 2003). However, when the direction of DNA replication conflicts with the direction of the tDNA transcription, it leads to replication-fork pausing (Osmundson et al., 2017; Yeung and Smith, 2020). Head-on replication-fork pausing promotes DNA damage by R-loop formation (Tran et al., 2017). R-loops are stable DNA:RNA hybrid structures with an unpaired DNA strand that naturally blocks replication but can also generate genomic instability (Santos-Pereira and Aguilera, 2015). If left unresolved, R-loops can create replication-transcription conflicts and lead to double-strand breaks which potentially increase DNA recombination (Hegazy et al., 2020). Tran et al. (2017) showed that tDNAs represent sites of double-strand breaks and of increased recombination events in a series of helicase mutants. Moreover, this phenomenon is also intimately connected with high expression levels of tRNAs and with the fact that tDNAs are usually associated with the pre-initiation complex, i.e., at a ready transcription state, which is a stable multiprotein complex consisting of a constant passage barrier for helicases (Arimbasseri et al., 2014).

Comparative genomics of 11 evolutionary-related yeast species showed a prevalence of tRNA genes at DNA breakpoints, which have also been linked to sites of genomic rearrangement (Gordon et al., 2009). One of the aspects that could underlie this observation is the preferential integration of transposable elements (TEs) at the proximity of tDNAs (Hani and Feldmann, 1998). TEs are mobile self-replicating elements that can integrate themselves in new genomic sites, being a potential source of mutations. There is an underlying assumption that TE insertions are deleterious, and indeed, they are a potential threat to genome integrity. S. cerevisiae has five families of TEs classified as long terminal repeat (LTR) retrotransposons, Ty1 to Ty5. The most abundant and active ones are the Ty1 and Ty2, which, apart from their ORFs, share a high sequence similarity between their LTR sequences (Carr et al., 2012). These long and near-identical sequences scattered in the genome are prone to recombination, particularly ectopic recombination, which allows for an array of rearrangements like deletions, duplications, inversions, and translocations (Mieczkowski et al., 2006). It is therefore important that a tight control of retrotransposons' expression is maintained. Tyl mobility is regulated by a retrograde mechanism where Ty1 self-encoded elements, like p22, inhibits Tyl's mobility when an elevated number of copies are present (Saha et al., 2015). This ability in S. cerevisiae was acquired by horizontal transfer from Saccharomyces paradoxus (Czaja et al., 2020). Nevertheless, comparative studies have shown that a large percentage of TEs are fixed in the genome (Bensasson, 2011), although there is also evidence for recent $T y$ insertions at a high rate (Carr et al., 2012), which can be seen as a source of genomic diversity and evolution. Increased retromobility has been observed upon exposure to several stress conditions like UV light (Bradshaw and McEntee, 1989) and adenine starvation (Todeschini et al., 2005). Furthermore, in physiological conditions like aging, retromobility has been observed in several species (Dennis et al., 2012; De Cecco et al., 2013; Li et al., 2013). In yeast, Maxwell et al. (2011) reported that during chronological aging, there is an association between Ty1 mobility and the observed genomic instability, particularly in loss of heterozygosity (LOH) events. Important adaptative roles for transposons have also been reported in experimentally evolved yeast. Dunham et al. (2002) studied the recombination events in evolved strains under glucose limitation and found that almost all detected rearrangements could be traced to ectopic rearrangement between transposons, transposon fragments, or tRNAs. In an experimental evolution study of cells expressing a mutant SertRNA (see below), there were large chromosomal rearrangements mediated by homologous recombination between transposons (Kalapis et al., 2015). In a large timescale, phylogenetic studies on genome evolution identified tRNAs and transposons at genome breakpoints and rearrangement sites (Fischer et al., 2000; Kellis et al., 2003; Gordon et al., 2009). Interestingly, in a comparative evolutionary study between $S$. cerevisiae and its related species S. paradoxus, S. mikatae, and S. uvarum, all the inversions identified were flanked by tDNAs in an opposite transcriptional orientation (Kellis et al., 2003). Thus, it is apparent that tDNAs, or their flaking regions, play an important role in genome innovation and evolution. Although it was not acknowledged in any study (at least to our understanding), it is possible that tDNAs (and their vicinity) represent "silent hotspots" for recombination that, when a particular condition is prolonged, become sites for "rapid" adaptive genomic alterations.

\section{GENOMIC CHANGES ASSOCIATED WITH TRNA MISEXPRESSION}

Upon environmental challenges, the organism quickly needs a particular set of defenses to survive. There is a body of evidence on how transcription changes in response to several stresses in yeast (Gasch et al., 2000; Gutin et al., 2019). It is, therefore, reasonable to assume that tRNA expression and abundance must also be tuned to follow these changes. Indeed, the tRNA pool dynamically changes to facilitate selective and faster translation of stress-related transcripts (Torrent et al., 2018). The tRNA pool is composed of various tRNA isoacceptor families, each encoded by tDNAs with different copy numbers. tRNA gene families with more copies of the same tDNA decode more frequently used codons, while tRNAs with one gene copy decode rarely used codons, which correlates with the codon usage of protein genes. This establishes the adequate balance between tRNA availability and the usage of its corresponding codon (Percudani et al., 1997). 
Curiously, not all copies of the same tRNA species contribute equally to the tRNA pool, and the loss of a particular copy can have different physiological consequences (Bloom-Ackermann et al., 2014). Thus, the multiplicity of copies enables higher expression of tRNAs in high translational demand and enables the tRNA pool to be dynamic enough to allow the dispensability of a particular copy to further expand the tRNA repertoire. This concept was explored by Yona et al. (2013) in a yeast strain with a deletion in the single-copy tDNA $t R(C C U) J$ (BloomAckermann et al., 2014), thus eliminating the only cognate tRNA for the AGG codon. Experimental evolution revealed that 200 generations were sufficient for cells to overcome the translational defect. Translational equilibrium was restored by mutating the anticodon of one of the 11 copies of Arg-tRNA $U C U$ from UCU

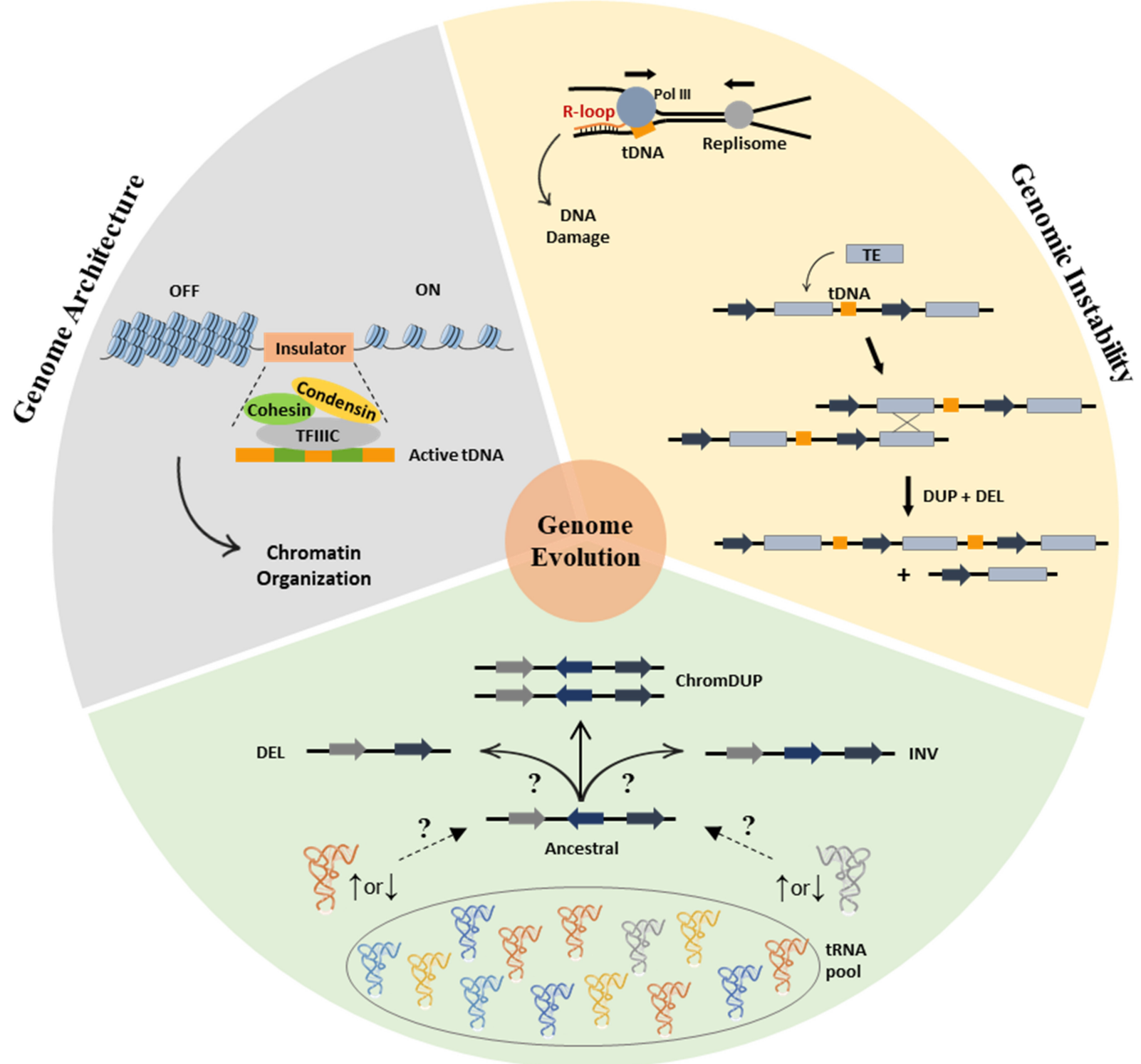

\section{Genomic Innovation}

FIGURE 1 | The involvement of tRNAs in genome organization and evolution. (Gray section) Role of tDNAs in genome architecture. TFIII recognizes the tDNA promoters (green boxes) enabling the assembly of the complete Pol III transcription apparatus and the recruitment of cohesin and condensin. Recruitment of the latter is crucial as it blocks the spread of heterochromatin (silenced state, OFF) into the active euchromatin (ON). (Yellow section) Contribution of tDNAs for genomic instability. tRNA genes are known sites for R-loop formation, which can be precursors of genomic instability, particularly when the directions of genome replication and of tRNA transcription collide (top). TEs integrate into the genome preferentially upstream of tDNAs. TEs are prone sites for ectopic recombination, like the one depicted, where recombination between sister chromatids results in deletions and duplications of the sequences located within the TEs (bottom). (Green section) The elusive connection between alterations in the tRNA pool and the genome instability. Alterations of tRNA's abundance level destabilize the proteome, leading to adaptive genome instability and mutations through poorly understood mechanisms (represented by "?"). 
to CCU, without affecting cellular fitness, highlighting how the plasticity of the tRNA pool can overcome translational challenges in changing environments.

Maintaining the proteome's good health is of extreme importance, but several bacterial and fungal species are able to decrease translation fidelity during stress to functionally diversify the proteome, a phenomenon called adaptive translation (Pan, 2013). Although alterations in the identity of a sense codon are a rare phenomenon, several budding yeasts reassigned the CUG codon to serine (Santos and Tuite, 1995) and to alanine (Muhlhausen et al., 2016; Riley et al., 2016). The CUG reassignments occurred independently during evolution and involved different tRNA genes that convergently mutated anticodons to CAG (Krassowski et al., 2018). Candida albicans is the most studied example of adaptive translation, where the identity of the CUG codon was altered to serine but residual leucine identity was still maintained. This results in an ambiguous CUG codon that is translated $97 \%$ of the times as serine and $3 \%$ as leucine, in standard growth conditions (Gomes et al., 2007). This is accomplished by a single Ser-tRNA ${ }_{C A G}$ with identity elements for both seryl- and leucyl-tRNA synthetases (Suzuki et al., 1997). However, alteration of the levels of the CUGdecoding tRNA is surprisingly adaptative. Bezerra et al. (2013) engineered a set of $C$. albicans strains with different combinations of tDNA $\mathrm{A}_{C A G}$ copy number, where one, two, or both copies of the endogenous Ser-tRNA ${ }_{C A G}$ genes were deleted and one or two copies of the $S$. cerevisiae Leu-tRNA ${ }_{C A G}$ genes were inserted, thus shifting the ratio of leucine/serine incorporated in the proteome. Strains tolerated increasing Leu incorporation and displayed unexpected phenotypic variability, with highly variable colony and cell morphologies, and increased tolerance to fluconazole and itraconazole. Interestingly, altering the copy number of the CUG decoding tDNAs leads to the rapid accumulation of unique single-nucleotide polymorphisms (SNPs) and $\mathrm{LOH}$ events. Strains with higher deregulation of the tRNA pool, and therefore higher levels of Leu incorporation at CUG sites, showed higher number of SNPs, indicating the potential mutagenic effect of tRNA codon misreading. Of note was the fact that strains with the most extreme alterations in the tRNA pool (i.e., with highest level of Leu incorporation) presented a near-complete $\mathrm{LOH}$ on chromosome V. A set of genes related with stress response, antifungal drug resistance, filamentous growth, and pathogenesis is located in this chromosome, showing that these alterations are not random and have an adaptative role (Bezerra et al., 2013). One could hypothesize that the observed genomic alterations triggered by tRNA misexpression are associated with the peculiar features of the C. albicans biology and its highly plastic genome (Selmecki et al., 2010). However, a similar phenotype was also uncovered in S. cerevisiae. Kalapis et al. (2015) experimentally evolved a yeast strain engineered with a mutant Ser-tRNA ${ }_{C A G}$ that misincorporates serine at CUG codons. Although this insertion was highly detrimental for fitness, cells were able to adapt to their new condition after 250 generations to their new condition. Genome sequencing showed that tolerance and adaptation to translational stress were achieved by large genomic rearrangements. These repeatedly involved a partial deletion of $127 \mathrm{~kb}$ at chromosome $\mathrm{V}$, enriched with genes involved in deubiquitination processes, and a duplication of $540 \mathrm{~kb}$ in chromosome IV, enriched with genes involved in glucose uptake. Together, these allowed cells to adapt to imbalances in the tRNA pool that culminate in CUG mistranslation by accelerated protein turnover and a high rate of glucose internalization (Kalapis et al., 2015). In other words, alterations in the tRNA pool and mRNA decoding accuracy destabilize the proteome in a dynamic way that reciprocates to the genome and produce important adaptive genome instabilities.

\section{CONCLUSION AND FUTURE PERSPECTIVES}

We highlighted the non-canonical function of tRNAs and tDNAs as drivers of genome evolution (Figure 1) and summarized how tDNA can play a role in the three-dimensional and functional organization of the genome and potentiate genome rearrangements events. Additionally, the discovery that alterations in the yeast tRNA pool generate genome instability associated with phenotypic variation of high adaptation potential adds a new dimension to the study of tRNA-driven genome evolution. Precisely how these mechanisms operate remains to be determined, but future work should elucidate how the tRNA pool provides evolutionary plasticity in environmental changing conditions. It is of high biological importance to understand the complex relationship between the tRNA pool and the genome, since the produced genomic instabilities may be relevant to human diseases, including cancer where extensive tRNA pool alterations and aneuploidies have been observed.

\section{AUTHOR CONTRIBUTIONS}

AG and AB: conceptualization, literature study, writing, and editing of the manuscript. MS: editing and revision of the manuscript. All authors listed have made a substantial, direct and intellectual contributions to the work, and approved it for publication.

\section{FUNDING}

This work was supported by FEDER (Fundo Europeu de Desenvolvimento Regional) funds through the COMPETE 2020, Operational Program for Competitiveness and Internationalization (POCI), and by Portuguese national funds via Fundação para a Ciência e a Tecnologia, I.P. (FCT) under the projects PTDC/BIA-MIB/31238/2017 and PTDC/BIA$\mathrm{MIC} / 31849 / 2017$. The $\mathrm{iBiMED}$ research unit was supported by FCT funds under UIDP/04501/2020. AG was supported directly by an FCT grant (SFRH/BD/121358/2016). AB was supported by national funds (OE), through FCT, I.P., in the scope of the framework contract foreseen in numbers 4,5 , and 6 of article 23, of Decree-Law 57/2016 of August 29, changed by Law $57 / 2017$ of July 19. 


\section{REFERENCES}

Arimbasseri, A. G., Rijal, K., and Maraia, R. J. (2014). Comparative overview of RNA polymerase II and III transcription cycles, with focus on RNA polymerase III termination and reinitiation. Transcription 5:e27639. doi: 10.4161/trns. 27369

Bagadia, M., Singh, A., and Singh Sandhu, K. (2016). Three dimensional organization of genome might have guided the dynamics of gene order evolution in eukaryotes. Genome Biol. Evol. 8, 946-954. doi: 10.1093/gbe/ evw050

Bensasson, D. (2011). Evidence for a high mutation rate at rapidly evolving yeast centromeres. BMC Evol. Biol. 11:211. doi: 10.1186/1471-2148-11-211

Bezerra, A. R., Simoes, J., Lee, W., Rung, J., Weil, T., Gut, I. G., et al. (2013). Reversion of a fungal genetic code alteration links proteome instability with genomic and phenotypic diversification. Proc. Natl. Acad. Sci. U.S.A. 110, 11079-11084. doi: 10.1073/pnas.1302094110

Biswas, M., Maqani, N., Rai, R., Kumaran, S. P., Iyer, K. R., Sendinc, E., et al. (2009). Limiting the extent of the RDN1 heterochromatin domain by a silencing barrier and Sir2 protein levels in Saccharomyces cerevisiae. Mol. Cell. Biol. 29, 2889-2898. doi: 10.1128/MCB.00728-08

Bloom-Ackermann, Z., Navon, S., Gingold, H., Towers, R., Pilpel, Y., and Dahan, O. (2014). A comprehensive tRNA deletion library unravels the genetic architecture of the tRNA pool. PLoS Genet. 10:e1004084. doi: 10.1371/journal. pgen.1004084

Bradshaw, V. A., and McEntee, K. (1989). DNA damage activates transcription and transposition of yeast Ty retrotransposons. Mol. Gen. Genet. 218, 465-474. doi: 10.1007/BF00332411

Carr, M., Bensasson, D., and Bergman, C. M. (2012). Evolutionary genomics of transposable elements in Saccharomyces cerevisiae. PLoS One 7:e50978. doi: 10.1371/journal.pone.0050978

Chan, P. P., and Lowe, T. M. (2016). GtRNAdb 2.0: an expanded database of transfer RNA genes identified in complete and draft genomes. Nucleic Acids Res. 44, D184-D189. doi: 10.1093/nar/gkv1309

Chan, Y. A., Aristizabal, M. J., Lu, P. Y., Luo, Z., Hamza, A., Kobor, M. S., et al. (2014). Genome-wide profiling of yeast DNA:RNA hybrid prone sites with DRIP-chip. PLoS Genet. 10:e1004288. doi: 10.1371/journal.pgen.1004288

Cole, H. A., Howard, B. H., and Clark, D. J. (2012). Genome-wide mapping of nucleosomes in yeast using paired-end sequencing. Methods Enzymol. 513, 145-168. doi: 10.1016/B978-0-12-391938-0.00006-9

Czaja, W., Bensasson, D., Ahn, H. W., Garfinkel, D. J., and Bergman, C. M. (2020). Evolution of Tyl copy number control in yeast by horizontal transfer and recombination. PLoS Genet. 16:e1008632. doi: 10.1371/journal.pgen.10 08632

D’Ambrosio, C., Schmidt, C. K., Katou, Y., Kelly, G., Itoh, T., Shirahige, K., et al. (2008). Identification of cis-acting sites for condensin loading onto budding yeast chromosomes. Genes Dev. 22, 2215-2227. doi: 10.1101/gad.1675708

De Cecco, M., Criscione, S. W., Peterson, A. L., Neretti, N., Sedivy, J. M., and Kreiling, J. A. (2013). Transposable elements become active and mobile in the genomes of aging mammalian somatic tissues. Aging 5, 867-883. doi: 10.18632/ aging. 100621

Dennis, S., Sheth, U., Feldman, J. L., English, K. A., and Priess, J. R. (2012). C. elegans germ cells show temperature and age-dependent expression of Cer1, a Gypsy/Ty3-related retrotransposon. PLoS Pathog. 8:e1002591. doi: 10.1371/ journal.ppat.1002591

Donze, D., Adams, C. R., Rine, J., and Kamakaka, R. T. (1999). The boundaries of the silenced HMR domain in Saccharomyces cerevisiae. Genes Dev. 13, 698-708. doi: $10.1101 /$ gad.13.6.698

Donze, D., and Kamakaka, R. T. (2001). RNA polymerase III and RNA polymerase II promoter complexes are heterochromatin barriers in Saccharomyces cerevisiae. EMBO J. 20, 520-531. doi: 10.1093/emboj/20.3.520

dos Reis, M., Savva, R., and Wernisch, L. (2004). Solving the riddle of codon usage preferences: a test for translational selection. Nucleic Acids Res. 32, 5036-5044. doi: 10.1093/nar/gkh834

Duan, Z., Andronescu, M., Schutz, K., McIlwain, S., Kim, Y. J., Lee, C., et al. (2010). A three-dimensional model of the yeast genome. Nature 465, 363-367. doi: 10.1038/nature08973

Dunham, M. J., Badrane, H., Ferea, T., Adams, J., Brown, P. O., Rosenzweig, F., et al. (2002). Characteristic genome rearrangements in experimental evolution of Saccharomyces cerevisiae. Proc. Natl. Acad. Sci. U.S.A. 99, 16144-16149. doi: 10.1073/pnas.242624799

El Hage, A., Webb, S., Kerr, A., and Tollervey, D. (2014). Genome-wide distribution of RNA-DNA hybrids identifies RNase $\mathrm{H}$ targets in tRNA genes, retrotransposons and mitochondria. PLoS Genet. 10:e1004716. doi: 10.1371/ journal.pgen.1004716

Fischer, G., James, S. A., Roberts, I. N., Oliver, S. G., and Louis, E. J. (2000). Chromosomal evolution in Saccharomyces. Nature 405, 451-454. doi: 10.1038/ 35013058

Gasch, A. P., Spellman, P. T., Kao, C. M., Carmel-Harel, O., Eisen, M. B., Storz, G., et al. (2000). Genomic expression programs in the response of yeast cells to environmental changes. Mol. Biol. Cell 11, 4241-4257. doi: 10.1091/mbc.11.12. 4241

Gomes, A. C., Miranda, I., Silva, R. M., Moura, G. R., Thomas, B., Akoulitchev, A., et al. (2007). A genetic code alteration generates a proteome of high diversity in the human pathogen Candida albicans. Genome Biol. 8:R206. doi: 10.1186/gb2007-8-10-r206

Gordon, J. L., Byrne, K. P., and Wolfe, K. H. (2009). Additions, losses, and rearrangements on the evolutionary route from a reconstructed ancestor to the modern Saccharomyces cerevisiae genome. PLoS Genet. 5:e1000485. doi: 10.1371/journal.pgen.1000485

Gutin, J., Joseph-Strauss, D., Sadeh, A., Shalom, E., and Friedman, N. (2019). Genetic screen of the yeast environmental stress response dynamics uncovers distinct regulatory phases. Mol. Syst. Biol. 15:e8939. doi: 10.15252/msb. 20198939

Haeusler, R. A., Pratt-Hyatt, M., Good, P. D., Gipson, T. A., and Engelke, D. R. (2008). Clustering of yeast tRNA genes is mediated by specific association of condensin with tRNA gene transcription complexes. Genes Dev. 22, 2204-2214. doi: $10.1101 /$ gad.1675908

Hamdani, O., Dhillon, N., Hsieh, T. S., Fujita, T., Ocampo, J., Kirkland, J. G., et al. (2019). tRNA genes affect chromosome structure and function via local effects. Mol. Cell. Biol. 39, e00432-18. doi: 10.1128/MCB.00432-18

Hani, J., and Feldmann, H. (1998). tRNA genes and retroelements in the yeast genome. Nucleic Acids Res. 26, 689-696. doi: 10.1093/nar/26.3.689

Hegazy, Y. A., Fernando, C. M., and Tran, E. J. (2020). The balancing act of R-loop biology: the good, the bad, and the ugly. J. Biol. Chem. 295, 905-913. doi: 10.1074/jbc.REV119.011353

Ivessa, A. S., Lenzmeier, B. A., Bessler, J. B., Goudsouzian, L. K., Schnakenberg, S. L., and Zakian, V. A. (2003). The Saccharomyces cerevisiae helicase Rrm3p facilitates replication past nonhistone protein-DNA complexes. Mol. Cell 12, 1525-1536. doi: 10.1016/s1097-2765(03)00456-8

Iwasaki, O., Tanaka, A., Tanizawa, H., Grewal, S. I., and Noma, K. (2010). Centromeric localization of dispersed Pol III genes in fission yeast. Mol. Biol. Cell 21, 254-265. doi: 10.1091/mbc.E09-09-0790

Kalapis, D., Bezerra, A. R., Farkas, Z., Horvath, P., Bodi, Z., Daraba, A., et al. (2015). Evolution of robustness to protein mistranslation by accelerated protein turnover. PLoS Biol. 13:e1002291. doi: 10.1371/journal.pbio.1002291

Kellis, M., Patterson, N., Endrizzi, M., Birren, B., and Lander, E. S. (2003). Sequencing and comparison of yeast species to identify genes and regulatory elements. Nature 423, 241-254. doi: 10.1038/nature01644

Krassowski, T., Coughlan, A. Y., Shen, X. X., Zhou, X., Kominek, J., Opulente, D. A., et al. (2018). Evolutionary instability of CUG-Leu in the genetic code of budding yeasts. Nat. Commun. 9:1887. doi: 10.1038/s41467-018-04374-7

LaBella, A. L., Opulente, D. A., Steenwyk, J. L., Hittinger, C. T., and Rokas, A. (2019). Variation and selection on codon usage bias across an entire subphylum. PLoS Genet. 15:e1008304. doi: 10.1371/journal.pgen.1008304

Li, W., Prazak, L., Chatterjee, N., Gruninger, S., Krug, L., Theodorou, D., et al. (2013). Activation of transposable elements during aging and neuronal decline in Drosophila. Nat. Neurosci. 16, 529-531. doi: 10.1038/nn.3368

Maxwell, P. H., Burhans, W. C., and Curcio, M. J. (2011). Retrotransposition is associated with genome instability during chronological aging. Proc. Natl. Acad. Sci. U.S.A. 108, 20376-20381. doi: 10.1073/pnas.1100271108

Mieczkowski, P. A., Lemoine, F. J., and Petes, T. D. (2006). Recombination between retrotransposons as a source of chromosome rearrangements in the yeast Saccharomyces cerevisiae. DNA Repair 5, 1010-1020. doi: 10.1016/j.dnarep. 2006.05.027

Muhlhausen, S., Findeisen, P., Plessmann, U., Urlaub, H., and Kollmar, M. (2016). A novel nuclear genetic code alteration in yeasts and the evolution of codon 
reassignment in eukaryotes. Genome. Res. 26, 945-955. doi: 10.1101/gr.2009 31.115

Noma, K., Cam, H. P., Maraia, R. J., and Grewal, S. I. (2006). A role for TFIIIC transcription factor complex in genome organization. Cell 125, 859-872. doi: 10.1016/j.cell.2006.04.028

Osmundson, J. S., Kumar, J., Yeung, R., and Smith, D. J. (2017). Pif1-family helicases cooperatively suppress widespread replication-fork arrest at tRNA genes. Nat. Struct. Mol. Biol. 24, 162-170. doi: 10.1038/nsmb.3342

Pan, T. (2013). Adaptive translation as a mechanism of stress response and adaptation. Annu. Rev. Genet. 47, 121-137. doi: 10.1146/annurev-genet111212-133522

Percudani, R., Pavesi, A., and Ottonello, S. (1997). Transfer RNA gene redundancy and translational selection in Saccharomyces cerevisiae. J. Mol. Biol. 268, $322-$ 330. doi: 10.1006/jmbi.1997.0942

Phizicky, E. M., and Hopper, A. K. (2010). tRNA biology charges to the front. Genes Dev. 24, 1832-1860. doi: 10.1101/gad.1956510

Pryce, D. W., Ramayah, S., Jaendling, A., and McFarlane, R. J. (2009). Recombination at DNA replication fork barriers is not universal and is differentially regulated by Swil. Proc. Natl. Acad. Sci. U.S.A. 106, 4770-4775. doi: 10.1073/pnas.0807739106

Raab, J. R., Chiu, J., Zhu, J., Katzman, S., Kurukuti, S., Wade, P. A., et al. (2012). Human tRNA genes function as chromatin insulators. EMBO J. 31, 330-350. doi: 10.1038/emboj.2011.406

Raina, M., and Ibba, M. (2014). tRNAs as regulators of biological processes. Front. Genet. 5:171. doi: 10.3389/fgene.2014.00171

Riley, R., Haridas, S., Wolfe, K. H., Lopes, M. R., Hittinger, C. T., Goker, M., et al. (2016). Comparative genomics of biotechnologically important yeasts. Proc. Natl. Acad. Sci. U.S.A. 113, 9882-9887. doi: 10.1073/pnas.16039 41113

Saha, A., Mitchell, J. A., Nishida, Y., Hildreth, J. E., Ariberre, J. A., Gilbert, W. V., et al. (2015). A trans-dominant form of Gag restricts Tyl retrotransposition and mediates copy number control. J. Virol. 89, 3922-3938. doi: 10.1128/JVI.03 060-14

Santos, M. A., and Tuite, M. F. (1995). The CUG codon is decoded in vivo as serine and not leucine in Candida albicans. Nucleic Acids Res. 23, 1481-1486. doi: $10.1093 /$ nar/23.9.1481

Santos-Pereira, J. M., and Aguilera, A. (2015). R loops: new modulators of genome dynamics and function. Nat. Rev. Genet. 16, 583-597. doi: 10.1038/nrg3961

Schramm, L., and Hernandez, N. (2002). Recruitment of RNA polymerase III to its target promoters. Genes Dev. 16, 2593-2620. doi: 10.1101/gad.1018902

Scott, K. C., Merrett, S. L., and Willard, H. F. (2006). A heterochromatin barrier partitions the fission yeast centromere into discrete chromatin domains. Curr. Biol. 16, 119-129. doi: 10.1016/j.cub.2005.11.065

Selmecki, A., Forche, A., and Berman, J. (2010). Genomic plasticity of the human fungal pathogen Candida albicans. Eukaryot Cell 9, 991-1008. doi: 10.1128/EC. 00060- 10

Simms, T. A., Dugas, S. L., Gremillion, J. C., Ibos, M. E., Dandurand, M. N., Toliver, T. T., et al. (2008). TFIIIC binding sites function as both heterochromatin barriers and chromatin insulators in Saccharomyces cerevisiae. Eukaryot Cell 7, 2078-2086. doi: 10.1128/EC.00128-08
Simms, T. A., Miller, E. C., Buisson, N. P., Jambunathan, N., and Donze, D. (2004). The Saccharomyces cerevisiae TRT2 tRNAThr gene upstream of STE6 is a barrier to repression in MATalpha cells and exerts a potential tRNA position effect in MATa cells. Nucleic Acids Res. 32, 5206-5213. doi: 10.1093/nar/gkh858

Su, Z., Wilson, B., Kumar, P., and Dutta, A. (2020). Noncanonical roles of tRNAs: tRNA fragments and beyond. Annu. Rev. Genet. 54, 47-69. doi: 10.1146/ annurev-genet-022620-101840

Suzuki, T., Ueda, T., and Watanabe, K. (1997). The 'polysemous' codon-a codon with multiple amino acid assignment caused by dual specificity of tRNA identity. EMBO J. 16, 1122-1134. doi: 10.1093/emboj/16.5.1122

Thompson, M., Haeusler, R. A., Good, P. D., and Engelke, D. R. (2003). Nucleolar clustering of dispersed tRNA genes. Science 302, 1399-1401. doi: 10.1126/ science. 1089814

Todeschini, A. L., Morillon, A., Springer, M., and Lesage, P. (2005). Severe adenine starvation activates Ty1 transcription and retrotransposition in Saccharomyces cerevisiae. Mol. Cell. Biol. 25, 7459-7472. doi: 10.1128/MCB.25.17.7459-7472. 2005

Torrent, M., Chalancon, G., de Groot, N. S., Wuster, A., and Madan Babu, M. (2018). Cells alter their tRNA abundance to selectively regulate protein synthesis during stress conditions. Sci. Signal. 11:eaat6409. doi: 10.1126/ scisignal.aat6409

Tran, P. L. T., Pohl, T. J., Chen, C. F., Chan, A., Pott, S., and Zakian, V. A. (2017). PIF1 family DNA helicases suppress R-loop mediated genome instability at tRNA genes. Nat. Commun. 8:15025. doi: 10.1038/ncomms15025

Wahba, L., Costantino, L., Tan, F. J., Zimmer, A., and Koshland, D. (2016). S1DRIP-seq identifies high expression and polyA tracts as major contributors to R-loop formation. Genes Dev. 30, 1327-1338. doi: 10.1101/gad.280834.116

Warner, J. R. (1999). The economics of ribosome biosynthesis in yeast. Trends Biochem. Sci. 24, 437-440. doi: 10.1016/s0968-0004(99)01460-7

Yeung, R., and Smith, D. J. (2020). Determinants of replication-fork pausing at tRNA genes in Saccharomyces cerevisiae. Genetics 214, 825-838. doi: 10.1534/ genetics. 120.303092

Yona, A. H., Bloom-Ackermann, Z., Frumkin, I., Hanson-Smith, V., CharpakAmikam, Y., Feng, Q., et al. (2013). tRNA genes rapidly change in evolution to meet novel translational demands. Elife 2:e01339. doi: 10.7554/eLife.01339

Yuan, G. C., Liu, Y. J., Dion, M. F., Slack, M. D., Wu, L. F., Altschuler, S. J., et al. (2005). Genome-scale identification of nucleosome positions in S. cerevisiae. Science 309, 626-630. doi: 10.1126/science.1112178

Conflict of Interest: The authors declare that the research was conducted in the absence of any commercial or financial relationships that could be construed as a potential conflict of interest.

Copyright (c) 2021 Guimarães, Correia, Sousa, Oliveira, Moura, Bezerra and Santos. This is an open-access article distributed under the terms of the Creative Commons Attribution License (CC BY). The use, distribution or reproduction in other forums is permitted, provided the original author(s) and the copyright owner(s) are credited and that the original publication in this journal is cited, in accordance with accepted academic practice. No use, distribution or reproduction is permitted which does not comply with these terms. 